\title{
Oferta de Carreras de Administración en los países de Centroamérica
}

\section{Offers Management Career in the countries of Central America}

\author{
Alex Medina Giacomozzi \\ Departamento Gestión Empresarial \\ Facultad De Ciencias Empresariales \\ Universidad Del Bío-Bío \\ Chillán, Chile \\ Alex@Ubiobio.Cl \\ Cecilia Gallegos Muñoz \\ Departamento Gestión Empresarial \\ Facultad De Ciencias Empresariales \\ Universidad Del Bío-Bio \\ Chillán, Chile \\ Cecilia@Ubiobio.Cl
}

Recibido: 20-VI-2011 • Aceptado 27-X-2011 • Corregido Corregido 20-XII-2011

Resumen: La educación es la base para el desarrollo de las personas, entre las cuales destaca la formación terciaria con su abanico de áreas formativas. Entre ellas está el área de Administración, la cual es parte de la oferta en las instituciones de educación superior de América Central, particularmente en los programas de segundo ciclo o grado, por lo que el objetivo de este articulo es exponer la variedad en la oferta de carreras en el área de Administración en Centroamérica, para asi aportar a un conocimiento más detallado sobre la variedad de esta oferta en cada país. La metodología implicó analizar las carreras de Administración de segundo ciclo de cada una de las instituciones de educación superior, por medio de la información pública emitida por medio de sus páginas web, extrayéndose de los currículos información sobre denominación, duración, salida intermedia, entre otros aspectos. Entre los resultados más significativos está el hecho que la gran mayoría de las carreras son presenciales y existen 325 programas distintos en 90 instituciones de educación superior. El título con mayor presencia es el de Administración de empresas y la certificación más utilizada es la de Licenciatura. La mayoría de los programas tiene una duración de cinco años.

\section{Introducción}

La educación es la base en la formación del capital humano, y en conjunto con la salud son un pilar fundamental para mejorar las condiciones de vida de la población; a partir de esta base, los países tendrán mejores opciones para su crecimiento y desarrollo, tal como lo plantea UNESCO (1998), y se permitirá la movilidad social de las personas.

La educación contempla en general tres niveles formativos, partiendo por un nivel primario o básico, seguido de un nivel secundario (también denominado colegial o medio) y un nivel superior o terciario. $\mathrm{Al}$ centrar el interés en esta educación terciaria, resaltan tres niveles: un primer nivel de formación técnica, un segundo nivel de bachiller o licenciatura (dependiendo del 
Palabras clave: Licenciatura en Administración, educación terciaria, certificación, universidades, carrera de grado en Administración

\begin{abstract}
Education is the basis for the development of people, among which tertiary education, with its range of training areas, including the administration area, which is part of the offer in institutions of higher education in Central America particularly in the second cycle programs or degree, so the aim of this paper is to present the variety in the provision of careers in management in Central America in order to provide a more detailed knowledge about the variety of this offer in each country. The methodology involved analyzing the careers of second cycle management of each institution of higher education through public information, issued through its web pages, extracting information about the curriculum name, duration, intermediate output, among other. Among the most significant is the fact that the vast majority of races are there in person and 325 different programs in 90 institutions of higher education. The title is the greater presence of business administration and certification most used is the degree. Most programs have duration of five years.
\end{abstract}

Keywords: Degree in business, tertiary education, certification, universities, undergraduate degree in administration país, puede uno u otro o ambos, como es el caso de Costa Rica) y un tercer nivel de postgrado (especialidad, magíster o doctorado), de los cuales es objeto de análisis en este estudio el segundo nivel de educación superior.

Es importante indicar que las instituciones de educación superior están integradas por universidades, institutos, escuelas, centros, etc., lo que hace muy variada a esta industria en cuanto al tipo de instituciones que participan en los distintos niveles de la educación superior.

Si bien la educación superior es muy relevante, el estudio acabado de carreras en particular no es un tema tratado profusamente en la literatura, lo más relacionado con esta investigación es el trabajo de Medina y Gallegos (2010) sobre la comparación de la formación del administrador por parte de las universidades en América Latina. También es importante el aporte de Fernández (1996) sobre el análisis comparado de 45 carreras de medicina en México, particularmente en lo que se refiere a la organización curricular, denominación, duración y otros aspectos relacionados; en el mismo sentido, está el artículo de Xue, Wu, Zhou, Yang y Story (2006), quienes realizan una comparación entre planes de estudio y estructura de programa de medicina entre una Universidad China y una australiana, aunque con un ámbito muy limitado.

Un estudio acotado a dos carreras y en dos universidades es el efectuado por Pirela y Peña (2006), quienes comparan el currículo de carreras de Bibliotecología y Archivología, en lo que se refiere a perfil y plan de estudios.

En el caso de Costa Rica, se relaciona en cierta medida a esta investigación el artículo de Chaverri y Sanabria (2010), quienes realizaron un estudio sobre la formación en Educación Básica, comparando universidades públicas con universidades privadas.

Dado lo anterior, resulta muy relevante direccionar la investigación sobre la carrera de Administración para el caso de América Central, lo que permite conocer 
con mayor claridad la oferta de este tipo de carreras en las instituciones de educación superior de Costa Rica, El Salvador, Guatemala, Honduras, Nicaragua y Panamá, las cuales, en su conjunto, representan el $66,3 \%$ del total de la población de Centroamérica, tal como se muestra en la figura 1.

Un antecedente importante es el total de alumnos en la educación superior en cada uno de estos países (tabla 1), destacándose
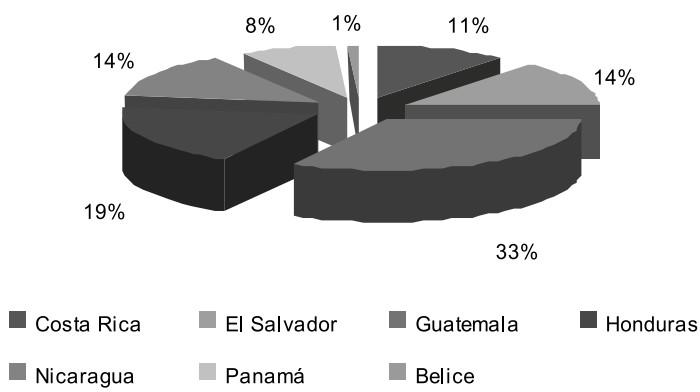

Figura $\mathrm{N}^{\circ}$ 1: Población de América Central

Fuente: Elaboración propia a partir de UNESCO (2008) en términos absolutos Panamá, pero al compararlo con el total de la población de cada país, la realidad es diversa, ya que, por un lado, la mayor cantidad de estudiantes en la educación superior se da en Panamá con un 3,78\%, seguido de Costa Rica con un 2,47\%. En un sentido contrario, la tasa más baja de participación está en Guatemala con un $0,86 \%$.

Considerando que la edad más habitual para cursar la educación superior fluctúa entre los dieciocho y veinticuatro años, resalta Panamá, pues un $44 \%$ de los jóvenes cursa algún programa de educación, seguido de Costa Rica con un 25\%. Es preocupante la situación de Guatemala, pues tiene solamente un $10 \%$ de los jóvenes en la educación superior, lo que indica que no cuenta con suficientes profesionales que le permitan contar con un capital humano en condiciones de enfrentar los desafíos del desarrollo.

Tabla 1

Instituciones de Educación Superior por país

\begin{tabular}{cccc}
\hline País & $\begin{array}{c}\text { No }^{\circ} \text { Alumnos } \\
\text { Educación Superior }\end{array}$ & \multicolumn{2}{c}{ Porcentaje Estudiantes } \\
\cline { 3 - 4 } & 110.716 & $\begin{array}{c}\text { Sobre total } \\
\text { población }\end{array}$ & $\begin{array}{c}\text { Sobre el total de } \\
\text { jóvenes }\end{array}$ \\
\hline Costa Rica & 122.431 & 1,72 & 25 \\
El Salvador & 114.767 & 0,86 & 19 \\
Guatemala & 112.874 & 1,57 & 10 \\
Honduras & 103.577 & 1,85 & 16 \\
Nicaragua & 126.242 & 3,78 & 18 \\
Panamá & & & 44 \\
\hline
\end{tabular}

Fuente: Elaboración propia a partir de UNESCO (2008)

En síntesis, esta investigación se centró en la oferta de segundo nivel y dentro de este nivel en la carrera de Administración. Para efectos de este estudio, se entiende por profesional de la Administración a aquella persona que posee un conjunto de competencias que se generan a partir del estudio de las Ciencias de la Administración y que está preparado para gestionar y que se espera que su desempeño profesional esté en el ámbito de la administración, tanto en el sector privado o público (Medina y Gallegos, 2010).

Por tanto, el objetivo de este artículo es exponer la variedad en la oferta de carreras publicadas en el área de Administración 
en Centroamérica por las instituciones de educación superior para así aportar a un conocimiento más detallado sobre la variedad de esta oferta en cada país.

Las instituciones de educación superior están conformadas por diversas entidades, tanto públicas como privadas, en donde se destacan en Centroamérica las universidades como las más representativas del sector, pero además se encuentran otras instituciones como institutos, centros o facultades.

\section{Metodología}

Esta investigación es un trabajo exploratorio de carácter cualitativo en donde se analizó la oferta de segundo nivel en las instituciones de educación superior en Costa Rica, El Salvador, Guatemala, Honduras, Nicaragua y Panamá, tanto de universidades como de otras instituciones a partir de la información oficial publicada por cada una de estas en su respectiva página web. Esto durante el segundo semestre de 2009, lo que contempló la revisión de los programas de estudio ofertados por 145 instituciones (ver anexo 1 con listado de instituciones), de las cuales se seleccionaron 90 instituciones que imparten programas en el área de Administración (figura 2). El análisis de la información se hizo mediante el uso de estadígrafos de tendencia central.

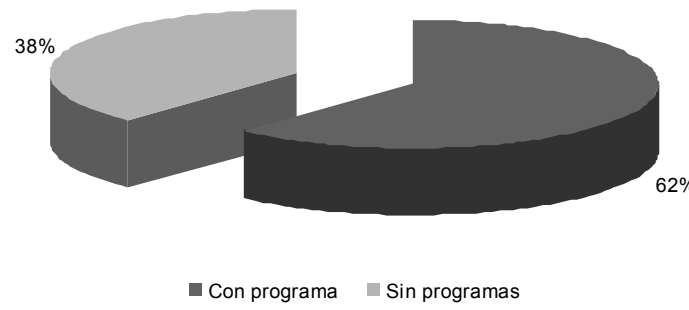

Figura $N^{\circ} 2$ : Instituciones de Educación Superior que ofrecen Carreras de Administración

Fuente: Elaboración propia
Estas 90 instituciones se separaron entre universidades y otras instituciones, además, cada programa se clasificó en generalista o especialista. Se entenderá por programa generalista aquella denominación amplia de programa, sin especificar ninguna área funcional de la administración o actividad económica a desarrollar, en sentido contrario, un programa especialista será aquel que en su denominación si contenga algún área funcional de la administración o actividad económica a realizar.

Una vez identificados los programas del área, se agruparon estos por tipos de denominación del programa, es decir, el nombre particular del título que recibirá el egresado o la egresada y, por otro lado, el tipo de certificación entregada, o sea, si este título es de licenciatura, bachiller, profesional u otro.

Además de lo anterior, se consideró la duración del programa en cada institución, bajo el criterio de años y clasificándolos según la cantidad de períodos anuales para obtener el respectivo título.

\section{Resultados}

De las 90 instituciones de los seis países analizados se tiene que 88 son universidades y 2 corresponden a otras instituciones, particularmente Instituto Tecnológico, las cuales ofrecen uno o más programas de segundo nivel en el área de administración, tal como se muestra en la tabla 2 .

De las 90 instituciones que tienen uno o más programas en el área de administración, la mayoría están en universidades, destacándose Nicaragua con 23 universidades que dictan programas en el área de Administración, seguido de Costa Rica (19 universidades). Honduras es el país con menos universidades que dictan esta especialidad. En el caso de Honduras y El Salvador, además de las universidades, también se ofrece programa en administración en institutos tecnológicos. 
Tabla 2

Instituciones con Programas en el área de Administración

\begin{tabular}{lllll}
\hline \multirow{2}{*}{ País } & \multirow{2}{*}{ Total } & \multicolumn{2}{c}{ Con programa } & Sin \\
\cline { 3 - 5 } & & Universidades & Institutos & programa \\
\hline \multicolumn{1}{c}{ Costa Rica } & 19 & 19 & 0 & 23 \\
El Salvador & 19 & 18 & 1 & 10 \\
Guatemala & 8 & 8 & 0 & 8 \\
Honduras & 5 & 4 & 1 & 2 \\
Nicaragua & 23 & 23 & 0 & 8 \\
Panamá & 16 & 16 & 0 & 4 \\
Totales & 90 & 88 & 2 & 55 \\
\hline
\end{tabular}

Fuente: Elaboración propia

Estas 90 instituciones de educación superior ofrecen un total de 325 programas en el área de Administración, tal como se expone en la tabla 3 , lo que claramente indica una oferta promedio de 3,6 programas por cada entidad.

Al analizar cada uno de estos programas, se clasificaron siguiendo la característica de su denominación, lo cual llevó a identificar, en términos generales a dos tipos de programas; por un lado, programas generalistas y, por otro lado, programas especialistas. De estos 325 programas se encontraron 78 programas generalistas y 247 programas especialistas.

Tabla 3

Número de programas por institución

\begin{tabular}{|c|c|c|c|c|c|c|c|}
\hline & \multirow{2}{*}{$\begin{array}{c}\mathrm{N}^{\circ} \\
\text { Programas }\end{array}$} & \multirow[t]{2}{*}{ Universidad } & \multirow[t]{2}{*}{ Institutos } & \multicolumn{2}{|c|}{ Universidad } & \multicolumn{2}{|c|}{ Institutos } \\
\hline & & & & Especialista & Generalista & Especialista & Generalista \\
\hline Costa Rica & 77 & 77 & 0 & 66 & 11 & 0 & 0 \\
\hline El Salvador & 38 & 37 & 1 & 17 & 20 & 1 & 0 \\
\hline Guatemala & 30 & 30 & 0 & 21 & 9 & 0 & 0 \\
\hline Honduras & 14 & 13 & 1 & 8 & 5 & 1 & 0 \\
\hline Nicaragua & 91 & 91 & 0 & 70 & 21 & 0 & 0 \\
\hline Panamá & 75 & 75 & 0 & 63 & 12 & 0 & 0 \\
\hline Totales & 325 & 323 & 2 & 245 & 78 & 2 & 0 \\
\hline
\end{tabular}

Fuente: Elaboración propia

El Salvador es el único país en donde prevalecen los programas generalistas por sobre los especialistas. Es notorio la fuerte presencia de programas especialistas en Nicaragua y Panamá.
Ahora bien, al hacer un análisis de conjunto se observa que los programas que tienen más presencia son los de carácter especialista ofrecidos por universidades, los cuales triplican a los programas generalistas 
dados por estas entidades. En el caso de los institutos, solamente existen dos programas y estos son especialistas.

Al centrar el interés en la denominación particular de cada uno de los programas se encontraron 179 diferentes denominaciones en instituciones que dictan uno o más programas en el área de administración, cuya variedad de nombres difiere de país en país, tal como se muestra en figura 3 (para mayor detalle, ver anexo 2)

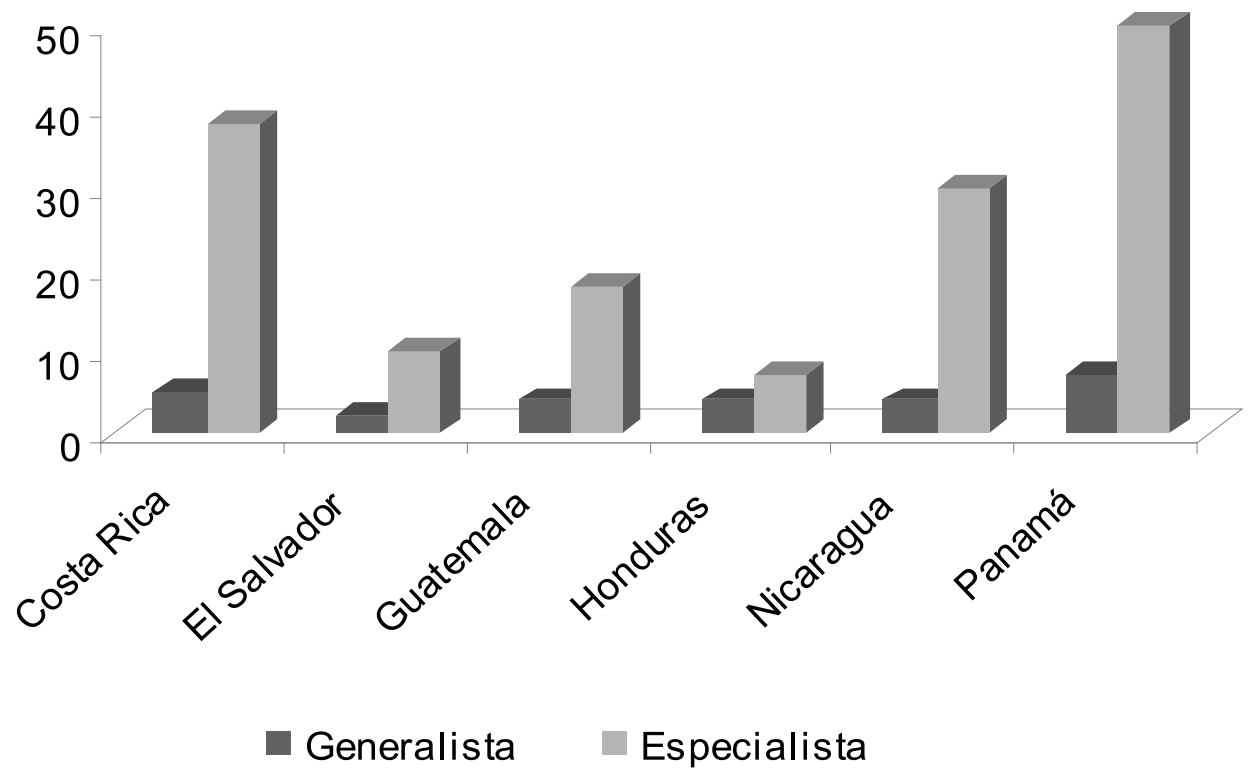

Figura $\mathrm{N}^{\circ} 3$ Denominación del Título por país Fuente: Elaboración propia

Llama la atención el caso de las instituciones de educación superior de Panamá, ya que presenta una muy alta variedad de denominaciones en los programas de segundo nivel en la educación terciaria en el área de Administración, no obstante no es el país con la mayor cantidad de instituciones y programas. En el caso de este país hay 57 denominaciones diferentes en los programas del área de administración.

Situación inversa a lo que ocurre en Panamá, es el caso de los programas ofertados por instituciones de educación superior de Honduras, existiendo solamente 11 denominaciones diferentes de programas en el área de Administración, ocurriendo que la mayor dispersión está en los programas especialistas ofertados por universidades.
En el caso de Costa Rica, presenta 43 denominaciones diferentes en los programas del área de Administración, en donde la gran mayoría de ellos es de carácter especialista. También es interesante la situación en Nicaragua, cuyas instituciones de educación superior ofertan 34 denominaciones diferentes en los programas del área de Administración, la mayoría especialistas.

Al profundizar en los tipos de denominación de los programas en el área de administración en los seis países bajo estudio, tal como se muestra en la figura 4, para el caso de las universidades, el nombre de programa generalista más utilizado es el de Administración de Empresas y con presencia en todos los países estudiados, en donde se destaca el caso de las 
universidades de El Salvador y Nicaragua. Las otras denominaciones no tienen una participación significativa, a excepción del programa en Administración de Negocios, el cual se oferta en Costa Rica y Panamá.

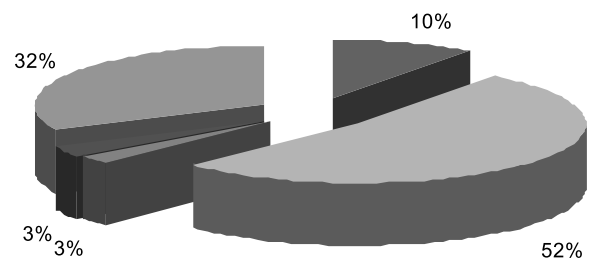

Administración de negocios

Administración de empresas

- Ingeniería comercial

Administración general

Otras denominaciones

Figura $\mathrm{N}^{\circ}$ 4: Programas Generalistas en Administración

Fuente: Elaboración propia

Adicional a lo anterior, al analizar los programas generalistas por país se tiene que en el caso de las universidades de Costa Rica, la denominación más utilizada es Administración de Negocios con un $45,45 \%$, nombre que también está presente en Panamá, con un 25\%. En lo que respecta a las universidades en El Salvador, la denominación más usada es Administración de Empresas, con un 95\%, lo que también se da en Guatemala, pero con un $66,67 \%$ y Nicaragua con un $80,95 \%$ de los programas.

También es destacable que la mayor dispersión de denominación de los programas en el área de Administración generalista en universidades está en Panamá y la mayor concentración en El Salvador.

En lo que respecta a los programas especialistas en universidades, hay una alta dispersión de denominaciones, tal como se muestra en el la figura 5, donde la mayor presencia es el programa de Mercadotecnia, ofrecido en universidades de cuatro de los seis países en estudio (El Salvador, Guatemala, Honduras y Nicaragua), al igual que Banca y Finanzas, el cual es dictado por universidades de Nicaragua y Panamá.
Al analizar por país las denominaciones de los programas especialistas, en Costa Rica el programa con mayor presencia es Administración de Empresas con énfasis en recursos humanos con sólo un 9\%; por su parte, para El Salvador, Guatemala y Honduras es Mercadotecnia con un $58,8 \%, 14,3 \%$ y $37,5 \%$, respectivamente. En el caso de Nicaragua y Panamá predomina Banca y Finanzas con un 14,3\% y $9,5 \%$, respectivamente.

En lo que respecta a la denominación de los programas del área de Administración especialista en los institutos, estos solamente tienen oferta en dos países, existiendo un programa de Economía y Negocios en el Salvador y un programa de Gestión en Agronegocios en Honduras.

En síntesis, al integrar todas las denominaciones de los programas ofertados por las distintas instituciones de cada país, tanto universidades como institutos, el programa con mayor oferta es Administración de Empresas, con 42 programas, seguido muy a lo lejos de Mercadotecnia y Banca y Finanzas, con 16 programas cada uno. Sin perjuicio de lo anterior, al integrar1 todas las denominaciones en el área específica de Mercadotecnia, se llega a un total de 35 programas, con lo que se concluye que son las denominaciones de programas más destacados.

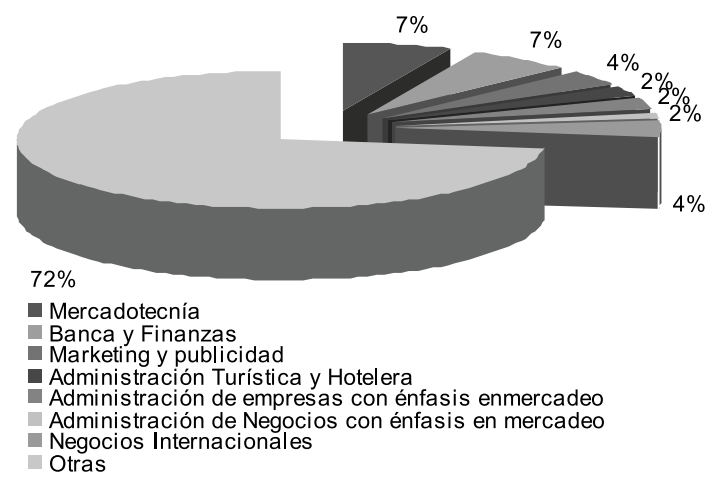

Figura $\mathrm{N}^{\circ}$ 5:Programas Generalistas en Administración

Fuente: Elaboración propia 
Una vez analizadas las diferentes denominaciones, también es relevante referirse al tipo de certificación empleada por cada una de las instituciones de educación superior de los países incluidos en la investigación. Tal como se muestra en la tabla
4, el tipo de certificación que prevalece es el de Licenciatura, el cual es otorgado por todos los países incluidos en la investigación. En El Salvador, Guatemala y Honduras se entrega, además, la certificación de Ingeniero.

Tabla 4

Tipo de Certificación

(número de programas por tipo de certificado)

\begin{tabular}{|c|c|c|c|c|}
\hline \multirow[t]{2}{*}{ País } & \multicolumn{2}{|c|}{ Licenciatura } & \multirow{2}{*}{$\begin{array}{c}\text { Ingeniero } \\
\text { Universidad }\end{array}$} & \multirow{2}{*}{$\begin{array}{c}\text { Sin } \\
\text { información }\end{array}$} \\
\hline & Universidad & Institutos & & \\
\hline Costa Rica & 77 & & & \\
\hline El Salvador & 34 & 1 & 1 & 2 \\
\hline Guatemala & 26 & & 2 & 2 \\
\hline Honduras & 12 & & 1 & 1 \\
\hline Nicaragua & 91 & & & \\
\hline Panamá & 68 & & & 7 \\
\hline
\end{tabular}

Fuente: Elaboración propia

Otro aspecto que también es importante exponer está referido a la duración de los diversos programas, tal como se muestra en la figura 6 , los cuales en su mayoría están entre 3 años (programas de 9 cuatrimestres) y 5 años (programas de 10 ciclos), siendo la duración más frecuente la de cuatro años, seguida de cinco años.

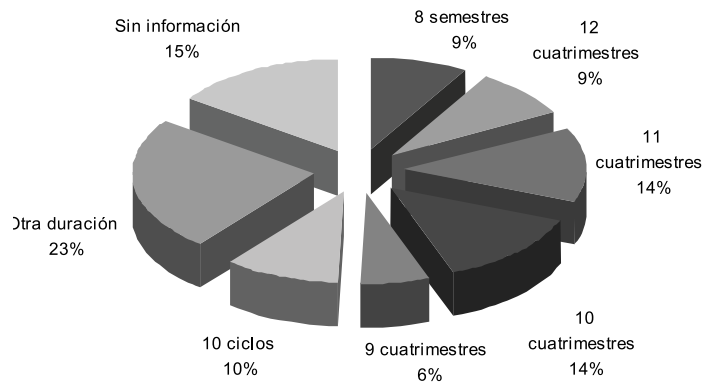

Figura $\mathrm{N}^{\circ}$ 6: Denominación del Título por país Fuente: Elaboración propia

Al observar la duración de los programas por cada país, en Costa Rica la duración más habitual es de 11 cuatrimestres (3,7 años), seguida de 10 cuatrimestres
(3,3 años). En El Salvador la duración es de 10 ciclos (5 años) en todos los programas y en Panamá la mayoría de los programas tienen una duración de 10 cuatrimestres (3,3 años) y de 9 cuatrimestres (3 años), para el caso de Guatemala la duración más común es de 8 semestres (4 años). En Honduras la duración más significativa es de 12 períodos (6 años) y en Nicaragua es de 12 cuatrimestres (4 años) y de 8 semestres (4 años).

Es destacable el caso de El Salvador, en el cual todos sus programas, independiente de la institución, tienen una duración de cinco años y todos medidos en ciclos.

Dada la duración de los programas, también es relevante relacionar esta duración con la modalidad de estudio que ofrece cada una de las instituciones investigadas. En este sentido, tal como se expone en el cuadro 5, la casi totalidad de estas tiene un plan de estudios que se debe cursar de forma presencial, conforme lo expuesto explícitamente en la información oficial expuesta en las páginas web institucionales. 
Tabla 5

Modalidad de estudios

\begin{tabular}{|c|c|c|c|}
\hline \multirow[t]{2}{*}{ País } & \multicolumn{2}{|c|}{ Presencial } & \multirow{2}{*}{$\begin{array}{c}\text { Semi } \\
\text { presencial }\end{array}$} \\
\hline & Universidad & Institutos & \\
\hline Costa Rica & 77 & & \\
\hline El Salvador & 36 & 1 & 1 \\
\hline Guatemala & 30 & & \\
\hline Honduras & 13 & 1 & \\
\hline Nicaragua & 91 & & \\
\hline Panamá & 75 & & \\
\hline
\end{tabular}

Fuente: Elaboración propia

Una vez expuesta la modalidad de estudios, se debe relacionar esta con el régimen de estudios, es decir, con la duración de cada período lectivo dentro de un año académico, así se encuentra con que en los países de América Central estudiados existe una gran variedad de regímenes de estudio para un mismo tipo de programa, tal como se expone en la figura 7 .

De acuerdo a la información recopilada, el régimen más común es el de cuatrimestre, el cual es utilizado en 147 programas, seguido del régimen de ciclo con 59 programas y el régimen semestral con 43 programas.

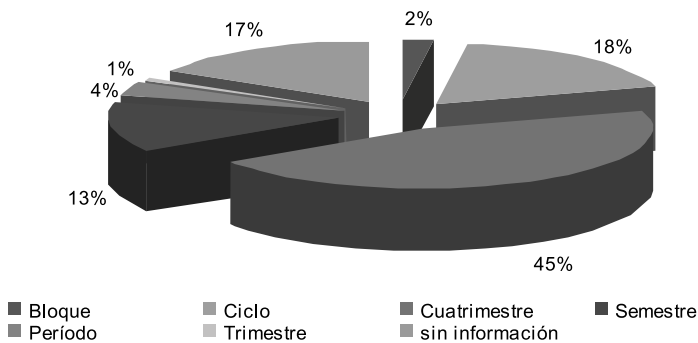

Figura $\mathrm{N}^{\circ} 7$ : Régimen de estudio

Fuente: Elaboración propia

Al analizar por país, en Costa Rica la mayoría de sus programas tienen un régimen cuatrimestral y en menor grado de ciclo y bloque. En El Salvador, todos los programas tienen un régimen de ciclo. En Guatemala, los programas se dividen principalmente entre ciclo y semestre. Para el caso de Honduras sus programas están en su gran mayoría en período. En Nicaragua, los programas se dividen de forma casi similar entre cuatrimestre y semestre. Panamá tiene marcada preferencia por el régimen cuatrimestral.

Por último, en relación a la salida intermedia o salida lateral, tal como se expone en la figura 8, de los diversos programas analizados, la mayoría de ellos no expone en su información oficial que contemple una salida intermedia.

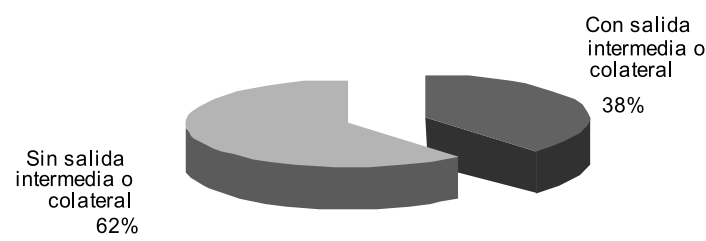

Figura $\mathrm{N}^{\circ}$ 8: Salida Intermedia o Colateral en los programas del área de Administración Fuente: Elaboración propia

Al analizar en particular las instituciones de educación superior se destacan Costa Rica y El Salvador pues todos sus programas cuentan con salida intermedia o lateral, a diferencia de Honduras cuyos programas no presentan información referente a la opción de salida intermedia. En cuanto al resto de los países 
bajo estudio, si bien algunos de ellos contemplan en su oferta una salida lateral, son una minoría en relación al total de programas ofrecidos (en Guatemala representan un $10 \%$, Nicaragua un $3 \%$ y Panamá un 5\%), que no lo indican.

En lo que respecta al tipo de certificación en la salida intermedia de las instituciones de educación superior que tienen esta opción, resalta con nitidez el título de Bachiller, tal como se muestra en la figura 9, el cual es otorgado exclusivamente por entidades de Costa Rica y El Salvador.

En cuanto al título de Técnico o Técnico superior como salida intermedia de los programas en el área de Administración, este es ofertado por entidades de Guatemala, Nicaragua y Panamá.

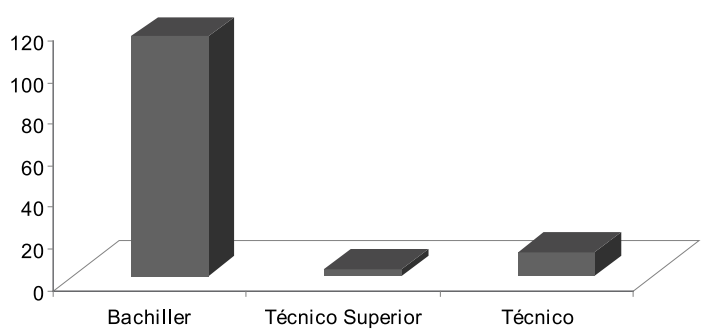

Figura $\mathrm{N}^{\circ}$ 9: Título Intermedio

Fuente: Elaboración propia

\section{Conclusión}

De los seis países analizados, solamente un $22 \%$ de las personas entre dieciocho y veinticuatro años está en la educación superior, lo que es una baja participación en la educación terciaria, comparado con América Latina que en su conjunto es de 31,5\%. La mejor cobertura está en Panamá con un $3,78 \%$ del total de personas en la educación superior y de un $44 \%$ de las personas entre dieciocho y veinticuatro años, seguido de Costa Rica con un $2,47 \%$ y $25 \%$, respectivamente. Situación contraria es la de Guatemala, pues solamente un $0,86 \%$ de la población y un $10 \%$ de las personas entre dieciocho y veinticuatro años están en la educación superior.

Del total de instituciones existentes en estos seis países, un $62 \%$ de ellas dicta un total de 325 programas en el área de Administración, lo que equivale, en promedio, a 3,6 programas por entidad.

De los 325 programas ofertados por las instituciones de educación superior, hay un $76 \%$ de programas especialistas, por lo que hay mayor incidencia de estos en Costa Rica, Guatemala, Honduras, Nicaragua y Panamá, excluyéndose El Salvador, en donde predominan los programas generalistas.

En cuanto a la denominación de estos programas hay 179 denominaciones diferentes en las instituciones que dictan uno o más programas en el área de Administración, al integrar estas, tanto universitarias como otras instituciones, se destaca el programa de Administración de Empresas, seguido por Mercadotecnia.

La certificación que prevalece es el de Licenciatura en todos los países y la mayoría de los programas tiene una duración de cinco años.

Es llamativo el caso de las instituciones de educación superior de El Salvador que ofrecen programas en el área de Administración, pues todos tienen una duración de cinco años, en régimen de ciclo y con salida lateral o intermedia, lo que indica un alto grado de concordancia, lo que favorece la movilidad estudiantil entre las instituciones.

También resalta el hecho que solamente una minoría de programas cuenta con salida intermedia o lateral y principalmente ofertados por instituciones de educación superior de Costa Rica y El Salvador.

Un aspecto que es importante destacar es que la difusión de carreras por medio de las páginas web es disímil entre instituciones de un mismo país y entre los países, existiendo instituciones con información muy completa y otras con una clara carencia de ella, lo cual afecta la comparación de 
un mismo programa entre instituciones, lo cual constituyó una limitación en el desarrollo de esta investigación.

Por último, este estudio demuestra la gran diversidad que existe en la oferta de carreras en el área de Administración en estos seis países y que es necesario seguir avanzando, por un lado, en incrementar el número de estudiantes en la educación superior y, por otro, en tratar de lograr mayores niveles de homologación entre los distintos programas del área.

\section{Notas}

Seintegraron los programas de Administración de Empresas con énfasis en mercadeo (6 programas), Mercadotecnia (16 programas), Administración de Negocios con énfasis en mercadeo (4 programas) y Marketing y Publicidad (9 programas).

\section{Anexos}

\section{Instituciones incluidas en la investigación}

\section{Costa Rica:}

Universidad de Costa Rica

Instituto Tecnológico de Costa Rica

Universidad Nacional

Universidad Estatal a Distancia

Universidad Autónoma de Centroamérica

Universidad Adventista

Universidad Panamericana

Universidad Latina Heredia

Universidad Hispanoariacana

Universidad Evangélica de las Américas

Universidad Braulio Carrillo

Universidad Autónoma de Monterrey

Universidad de la Salle

Universidad de Cartago Florencio del Castillo

Universidad Isaac Newton

Universidad Santa Lucía

Universidad Magister

Universidad Juan Pablo II

Universidad Metropolitana Castro Carazo

Universidad de las Ciencias y el Arte de Costa Rica

Universidad Americana

Universidad Central

Universidad San José
Universidad Libre de Costa Rica

Universidad Veritas

Universidad Fidelitas

Universidad Federada de Costa Rica

Universidad de Ciencias Administrativas San Marcos

Universidad San Juan de la Cruz

Universidad Independiente de Costa Rica

Universidad Internacional San Isidro Labrador

Universidad Cristiana del Sur

Universidad Tecnológica Costarricense

Universidad Cristiana Internacional

Universidad Metodista

Universidad Católica de Costa Rica

Universidad Fundepos Alma Mater

Universidad Creativa

Universidad Internacional de las Américas

Universidad Latinoamericana de Ciencia y Tecnología

Universidad Latina de Costa Rica

Universidad del Valle

\section{El Salvador}

Universidad de El Salvador

Universidad Albert Einstein

Universidad Autónoma de Santa Ana

Universidad Capitán General Gerardo Barrios

Universidad Católica de El Salvador

Universidad Centroamericana José Simeón Cañas

Universidad de Oriente

Universidad de Sonsonate

Universidad Don Bosco

Universidad Dr. Andrés Bello

Universidad Dr. José Matías Delgado

Universidad Evangélica de El Salvador

Universidad Francisco Gavidia

Universidad Luterana Salvadoreña

Universidad Modular Abierta

Universidad Monseñor Oscar Arnulfo Romero

Universidad Nueva San Salvador

Universidad Panamericana

Universidad Pedagógica de El Salvador

Universidad Salvadoreña Alberto Masferrer

Universidad Técnica Latinoamericana

Universidad Tecnológica de El Salvador

Universidad Politécnica de El Salvador

Universidad Cristiana de la Asamblea de Dios

Universidad Leonardo Da Vinci

Universidad Salvadoreña Isaac Newton

Instituto Tecnológico Centroamericano

Universidad Católica de Occidente

Universidad Panamericana de El Salvador

\section{Nicaragua:}

Universidad Nacional Autónoma de Nicaragua Universidad Nacional de Ingeniería

Universidad Centroamericana UCA - Nicaragua

Universidad Politécnica de Nicaragua

Universidad Central de Nicaragua

Universidad Americana 
Universidad Thomas More

Universidad de Ciencias Comerciales

Universidad del Valle

Universidad de Managua

Universidad de las Américas

Universidad Jean Jacques Rousseau

Universidad Paulo Freire

Universidad Popular de Nicaragua

Universidad Técnica de Comercio

Universidad Juan Pablo II

Universidad Martín Lutero (UML)

Universidad Nicaragüense de Estudios Humanísticos

Universidad del Norte de Nicaragua

Universidad de Tecnología y Comercio

Universidad Católica "Redemptoris Mater"

Universidad Nicaragüense de Ciencia y Tecnología

Universidad Iberoamericana de Ciencia y Tecnología

Universidad Evangélica Nicaragüense "Martín

Luther King Jr."

Universidad Hispanoamericana

Universidad Internacional para el Desarrollo Sostenible

Universidad Internacional de la Integración de

América Latina

Universidad Nacional Autónoma de Nicaragua León

Universidad Cristiana Autónoma de Nicaragua

Universidad Internacional de la Integración de

América Latina

Universidad de las Regiones Autónomas de la Costa

Caribe Nicaragua

\section{Panamá:}

Universidad de Panamá

Universidad Tecnológica de Panamá

Universidad ISAE

Universidad Especializada de Las Américas

Universidad Latina de Panamá

Universidad Autónoma de Chiriquí

Columbus University

Universidad del Istmo

Universidad de Cartago

Universidad Tecnológica Oteima

Universidad Internacional de Comercio y Educación

Universidad Americana de Panamá

Universidad Metropolitana de Educación Ciencias. y

Tecnología

International University

Laureate International Universities

Universidad Latinoamericana de Comercio Exterior

Universidad Marítima Internacional de Panamá

Aden Business School Universidad Alta Dirección

Universidad Abierta y a distancia de Panamá

Universidad Católica Santa María la Antigua USMA

Panamá

\section{Guatemala:}

Facultad Latinoamericana de Ciencias Sociales FLACSO

Universidad Mariano Gálvez de Guatemala
Universidad Rafael Landivar

Universidad Mesoamericana Guatemala

Universidad Panamericana de Guatemala

Universidad Rural de Guatemala

Universidad InterNaciones

Universidad San Pablo de Guatemala

Universidad del Istmo

Universidad del Valle de Guatemala

Universidad Francisco Marroquín

Centro Universitario de Occidente

Centro Universitario Ciudad Vieja

Centro Universitario de Oriente

Universidad de San Carlos de Guatemala

Universidad Galileo

\section{Honduras:}

Universidad Tecnológica Centroamericana

Universidad Metropolitana de Honduras

Universidad Tecnológica de Honduras

Universidad Politécnica de Ingeniería de Honduras

Universidad Nacional Autónoma de Honduras

Universidad Católica de Honduras

Universidad de San Pedro Sula

Instituto Tecnológico Sampedrano

\section{Denominación de programas más utilizados}

-Administración de Negocios

-Administración de Empresas

- Ingeniería Comercial

-Administración Gerencial

-Administración de Empresas con énfasis en recursos humanos

-Administración de Empresas con énfasis en mercadeo

- Mercadotecnia

-Administración de Negocios énfasis en mercadeo

- Banca y Finanzas

- Marketing y Publicidad

-Administración Turística y Hotelera

- Comercio internacional

- Negocios internacionales

-Administración de Recursos Humanos

-Administración de Oficinas

-Administración de Empresas con concentración en contabilidad

- Gerencia

-Administración de Empresas con concentración en gerencia

-Administración Turística

-Administración Hotelera

-Economía y Negocios

-Administración de Empresas con concentración en banca

-Administración de Sistemas de oficina

-Administración de Empresas con concentración en sistemas de información 
- Mercadeo

- Gestión de Agronegocios

- Otras Denominaciones

\section{Referencias bibliográficas}

Aguado, S. (2002). La gestión innovadora. Recuperado de http://www.solveidaaguad19.@hotmail.com

Aden Business School Universidad Alta Dirección, Recuperado de http:// www.aden.org

Centro Universitario de Oriente, Guatemala. Recuperado de http:// www. cunori.edu.gt

Centro Universitario de Occidente, Guatemala. Recuperado de http:// www.cunoc.usac.edu.gt

Centro Universitario Ciudad Vieja, Guatemala. Recuperado de http:// www.cucv.edu.gt

Chaverri, D. y Sanabria, M. (2010): "Estudio comparativo entre los programas que ofrecen tres universidades públicas y siete universidades privadas en la carrera de I y II ciclo de la Educación General Básica en Costa Rica". Revista Actualidades Investigativas en Educación, 10(13), 1-33, San José.

Columbus University. Recuperado de http:// www.columbus.edu.pa

Facultad Latinoamericana de Ciencias Sociales - FLACSO, Guatemala. Recuperado de http://www.flacso.edu. gt

Fernández, J. (1996): "Las carreras de medicina en México". Revista Perfiles Educativos, XVIII(73), julio-septiembre, México

Instituto Tecnológico Centroamericano, El Salvador, Recuperado de http://www. itca.edu.sv

Instituto Tecnológico de Costa Rica, Costa Rica. Recuperado de http:// www.tec.ac.cr

Instituto Tecnológico Sampedrano, Honduras. Recuperado de http://www.itshm.com
International University. Recuperado de http://www.interuni.us

Laureate International Universities. Recuperado de http://www.ulacit.ac.pa

Medina, A. Y Gallegos, C. (2010): "Formación del profesional de la administración en América Latina: estudio comparado", Revista Educación y Educadores , Vol 13, $N^{\circ}$ 2, Bogotá

Pirela, J. y Peña, T. (2006): "La formación del profesional de la información en Venezuela: Una mirada comparativa desde sus diseños curriculares". Educer-Investigaciones Arbitrales, 10(32), enero-marzo, 131-138.

Real Academia de la Lengua (2010): "Diccionario de la lengua española", Editorial Espasa Calpe. Recuperado de http://www.espasa.es/actualizacion_rae/drae.html

UNESCO (2008): "Global education digest 2007; Comparing education statistics across World", Editado por Unesco.

UNESCO (1998): "Declaración mundial sobre la educación superior en el siglo XXI: Visión y acción”. Recuperado de http://www.unesco. org/education/educprog/wche/declaration_spa.htm

Universidad del Istmo. Recuperado de http://www.udi.edu.pa

Universidad de Cartago. Recuperado de http://www.ucapanama.org

Universidad Tecnológica Oteima. Recuperado de: http://www.oteima.ac.pa

Universidad Mariano Gálvez de Guatemala, Guatemala. Recuperado de http:// www.umg.edu.gt

Universidad Rafael Landivar, Guatemala. Recuperado de http://www.url.edu.gt

Universidad Abierta y a distancia de Panamá. Recuperado de http://www. unadp.ac.pa

Universidad Adventista, Costa Rica. Recuperado de http://www.unadeca.net

Universidad Albert Einstein, El Salvador. Recuperado de http://wwwuae.edu.sv.

Universidad Americana de Panamá. Recuperado de http://www.uam.ac.pa 
Universidad Americana, Costa Rica. Recuperado de http://www.uam.ac.cr

Universidad Americana, Nicaragua. Recuperado de http://www.uam.edu.ni

Universidad Thomas More, Nicaragua. Recuperado de http://www.unithomasmore.edu.ni

Universidad de Ciencias Comerciales, Nicaragua. Recuperado de http:// www.ucc.edu.ni

Universidad del Valle, Nicaragua. Recuperado de http://www.univalle.edu.ni

Universidad de Managua, Nicaragua. Recuperado de http://www.udem.edu.ni

Universidad Autónoma de Centroamérica. Recuperado de http://www.uaca.ac.cr

Universidad Autónoma de Chiriquí. Recuperado de http://www.unachi. ac.pa

Universidad Autónoma de Monterrey, Costa Rica. Recuperado de http:// www.unamon.com

Universidad Autónoma de Santa Ana, El Salvador. Recuperado de http://www. unasa.edu.sv

Universidad Braulio Carrillo, Costa Rica. Recuperado de http://www.universidadbrauliocarrillo.com

Universidad Capitán General Gerardo Barrios, El Salvador. Recuperado de http://www.ugb.edu.sv

Universidad Católica de Costa Rica, Costa Rica. Recuperado de http://www.ucatolica.ac.cr

Universidad Católica de El Salvador, El Salvador. Recuperado de http://www. catolica.edu.sv

Universidad Católica de Honduras, Honduras. Recuperado de http:// www.unicah.edu.hn

Universidad Católica de Occidente,El Salvador. Recuperado de http://www. unic.edu.sv

Universidad Católica Santa María la Antigua USMA Panamá. Recuperado de http://www.usma.ac.pa

Universidad Central, Costa Rica. Recuperado de http://www.universidadcentral.com
Universidad Centroamericana José Simeón Cañas, El Salvador. Recuperado de http://www.uca.edu.sv

Universidad Creativa, Costa Rica. Recuperado de http://www.ucreativa.com

Universidad Cristiana Autónoma de Nicaragua, Nicaragua. Recuperado de http://www.ucan.edu.ni

Universidad Internacional de la Integración de América Latina, Nicaragua. Recuperado de http:// www.unival.edu.ni

Universidad Cristiana de la Asamblea de Dios, El Salvador. Recuperado de http://www.ucad.edu.sv

Universidad Cristiana del Sur, Costa Rica. Recuperado de http://www.scu.ac.cr

Universidad Cristiana Internacional, Costa Rica. Recuperado de http:// www.esepa.org

Universidad de Cartago Florencio del Castillo, Costa Rica. Recuperado de http://www.uca.ac.cr

Universidad de Ciencias Administrativas San Marcos, Costa Rica. Recuperado de http://www.usam.ac.cr

Universidad de Costa Rica, Costa Rica, Recuperado de http://www.ucr.ac.cr

Universidad de El Salvador, El Salvador. Recuperado de http://www.ues.edu.sv Universidad de las Regiones Autónomas de la Costa Universidad de la Salle, Costa Rica. Recuperado de http:// www.ulasalle.ac.cr

Universidad de las Américas, Nicaragua. Recuperado de http://www.ulam.edu.ni Universidad de las Ciencias y el Arte de Costa Rica, Costa Rica. Recuperado de http://www.udelascienciasyelarte.ac.cr

Caribe Nicaragua, Nicaragua. Recuperado de http://www.uraccan.edu.ni

Universidad de Oriente, El Salvador. Recuperado de http://www.univo. edu.sv

Universidad de Panamá, Panamá. Recuperado de http://www.up.ac.pa

Universidad de San Carlos de Guatemala, Guatemala. Recuperado de http:// www.usac.edu.gt 
Universidad de San Pedro Sula, Honduras, Recuperado de http://www.usps.edu.hn

Universidad de Sonsonate, El Salvador. Recuperado de http://www.usonsonate.edu.sv

Universidad de Tecnología y Comercio, Nicaragua. Recuperado de http:// www. unitec.edu.ni

Universidad Católica "Redemptoris Mater", Nicaragua. Recuperado de http:// www.unica.edu.ni

Universidad Nicaragüense de Ciencia y Tecnología, Nicaragua. Recuperado de http://www.ucyt.edu.ni

Universidad Iberoamericana de Ciencia y Tecnología, Nicaragua. Recuperado de http://www.unicit.edu.ni

Universidad Evangélica Nicaragüense "Martín Luther King Jr." Nicaragua. Recuperado de http:// www.uenicmlk.edu.ni

Universidad Hispanoamericana, Nicaragua. Recuperado de http:// www.uhispam.edu.ni

Universidad Internacional para el Desarrollo Sostenible, Nicaragua. Recuperado de http://www.unides.edu.ni

Universidad Internacional de la Integración de América Latina, Nicaragua. Recuperado de http:// www.unival.edu.ni

Universidad Nacional Autónoma de Nicaragua León, Nicaragua. Recuperado de http://www.unanleon.edu.ni

Universidad del Norte de Nicaragua, Nicaragua. Recuperado de http:// www.unnnicaragua.org

Universidad del Valle, Costa Rica. Recuperado de http://www.udelvalle. com

Universidad Don Bosco, El Salvador. Recuperado de http://www.udb.edu.sv

Universidad Dr. Andrés Bello, El Salvador, Recuperado de http:// www.unab.edu.sv

Universidad Dr. José Matías Delgado, El Salvador. Recuperado de http:// www.ujmd.edu.sv
Universidad Especializada de Las Américas. Recuperado de http://www.udelas.ac.pa Universidad Estatal a Distancia, Costa Rica, Recuperado de http://www.uned.ac.cr Universidad Evangélica de El Salvador, El Salvador, Recuperado de http://www. uees.edu.sv

Universidad Evangélica de las Américas, Costa Rica, Recuperado de http:// www.unela.ac.cr

Universidad Federada de Costa Rica, Costa Rica, Recuperado de http://www.ufederada.ac.cr

Universidad Fidelitas, Costa Rica. Recuperado de http://www.ufidelitas.ac.cr

Universidad Francisco Gavidia, El Salvador. Recuperado de http://www.ufg.edu.sv Universidad Fundepos Alma Mater, Costa Rica, Recuperado de http://www.fundepos.ac.cr

Universidad Galileo, Guatemala. Recuperado de http://www.galileo. edu.gt

Universidad Hispanoamericana, Costa Rica. Recuperado de http://www. Uhispanoamericana.ac.cr

Universidad Independiente de Costa Rica, Costa Rica, Recuperado de http:// www.uindependiente.ac.cr

Universidad Internacional de Comercio y Educación. Recuperado de http:// www.uniced.ac.pa

Universidad Internacional de las Américas, Costa Rica. Recuperado de http:// www.uia.ac.cr

Universidad Internacional San Isidro Labrador, Costa Rica. Recuperado de http://www.uisil.com

Universidad Isaac Newton, Costa Rica, Recuperado de http://www.unin.ac.cr Universidad ISAE, Recuperado de http:// www.isaeuniversidad.com

Universidad Jean Jacques Rousseau, Nicaragua. Recuperado de http:// www.unijjar.edu.ni

Universidad Paulo Freire, Nicaragua. Recuperado de http://www.upf.edu.ni Universidad Popular de Nicaragua, 
Nicaragua. Recuperado de http:// www.uponic.edu.ni

Universidad Técnica de Comercio, Nicaragua. Recuperado de http:// www.utc.edu.ni

Universidad Juan Pablo II, Nicaragua. Recuperado dehttp://www.univjuanpablo2.com

Universidad Martín Lutero (UML) , Nicaragua. Recuperado dehttp:// www.uml.edu.ni

Universidad Nicaragüense de Estudios Humanísticos, Nicaragua. Recuperado de http://www.uneh.es.tl

Universidad Juan Pablo II, Costa Rica, Recuperado de http://www.ujpil.ac.cr

Universidad Latina de Costa Rica, Costa Rica. Recuperado dehttp://www.ulatina.ac.cr

Universidad Latina de Panamá. Recuperado de http://www.ulat.ac.pa

Universidad Latina Heredia, Costa Rica. Recuperado de http://www.ulatina. ac.cr

Universidad Latinoamericana de Ciencia y Tecnología, Costa Rica. Recuperado de http://www.ulacit.ac.cr

Universidad Latinoamericana de Comercio Exterior. Recuperado de http://www. ulacex.com

Universidad Marítima Internacional de Panamá. Recuperado de http://www. umip.ac.pa

Universidad Leonardo Da Vinci, El Salvador. Recuperado de http://www. davinci.edu.sv

Universidad Libre de Costa Rica, Costa Rica, Recuperado dehttp://www.ulicori.ac.cr

Universidad Luterana Salvadoreña, El Salvador. Recuperado de http:// www.uls.edu.sv

Universidad Magister, Costa Rica, Recuperado de http://www.umagister.com

Universidad Mesoamericana Guatemala, Guatemala. Recuperado de http:// www.umes.edu.gt

Universidad Panamericana de Guatemala, Guatemala. Recuperado de http:// www.upana.edu.gt
Universidad Rural de Guatemala, Guatemala. Recuperado de http:// www.urural.edu.gt

Universidad InterNaciones, Guatemala, Recuperado de http://www.uni.edu.gt

Universidad Metodista, Costa Rica. Recuperado de http://www.unimet. ac.cr

Universidad Metropolitana Castro Carazo, Costa Rica. Recuperado de http:// www.umca.net

Universidad Metropolitana de Educación Ciencias. y Tecnología. Recuperado de http://www. Umecit.edu.pa

Universidad Modular Abierta, El Salvador. Recuperado de http:// www.uma.edu.sv

Universidad Monseñor Oscar Arnulfo Romero, El Salvador, Recuperado de http://www.umoar.edu.sv

Universidad Nacional Autónoma de Honduras, Honduras. Recuperado de http://www.unah.edu.hn

Universidad Nacional Autónoma de Nicaragua, Nicaragua. Recuperado de http://www.unan.edu.ni

Universidad Nacional de Ingeniería, Nicaragua. Recuperado de http:// www.uni.edu.ni

Universidad Centroamericana UCA Nicaragua, Nicaragua. Recuperado de http://www.uca.edu.ni

Universidad Politécnica de Nicaragua, Nicaragua. Recuperado de http:// www.upoli.edu.ni

Universidad Central de Nicaragua, Nicaragua. Recuperado de http:// www.ucn.edu.ni

Universidad Nacional, Recuperado de http://www.una.ac.cr

Universidad Nueva San Salvador, El Salvador. Recuperado de http://www. unssa.edu.sv

Universidad Panamericana de El Salvador, El Salvador. Recuperado de http:// www.upan.edu.sv

Universidad Panamericana, Costa Rica. Recuperado de http://www.upanamericana.net 
Universidad Panamericana, El Salvador. Recuperado de http://www.upan. edu.sv

Universidad Pedagógica de El Salvador, El Salvador. Recuperado de http://www. pedagogica.edu.sv

Universidad Politécnica de El Salvador, El Salvador, Recuperado de http://www. upes.edu.sv

Universidad Salvadoreña Alberto Masferrer, El Salvador. Recuperado de http://www.usam.edu.sv

Universidad Salvadoreña Isaac Newton, El Salvador. Recuperado de http://www. usin.edu.sv

Universidad San José, Costa Rica. Recuperado de http://www.usanjose.ac.cr

Universidad San Juan de la Cruz, Costa Rica, Recuperado de http://www.sjdlc.cr

Universidad San Pablo de Guatemala, Guatemala. Recuperado dehttp:// www.uspg.edu.gt

Universidad del Istmo, Guatemala. Recuperado de http://www.unis.edu.gt

Universidad del Valle de Guatemala, Guatemala. Recuperado dehttp:// www.uvg.edu.gt

Universidad Francisco Marroquín, Guatemala. Recuperado de http:// www.ufm.edu.gt

Universidad Politécnica de Ingeniería de Honduras, Honduras, Recuperado de http://www.upi.edu.hn
Universidad Santa Lucía, Costa Rica, Recuperado de http://www.usantalucia.com

Universidad Técnica Latinoamericana, El Salvador. Recuperado de http://www. utla.edu.sv

Universidad Tecnológica Centroamericana, Honduras. Recuperado de http:// www.unitec.edu.hn

Universidad Metropolitana de Honduras, Honduras. Recuperado de http:// www.unimetro.edu.hn

Universidad Tecnológica Costarricense, Costa Rica. Recuperado de http:// www.utccr.com

Universidad Tecnológica de El Salvador, El Salvador. Recuperado de http://www. utec.edu.sv

Universidad Tecnológica de Honduras, Honduras. Recuperado de http:// www.uth.edu.hn

Universidad Tecnológica de Panamá, Panamá. Recuperado de http:// www.utp.ac.pa

Universidad Veritas, Costa Rica. Recuperado de http://www.uveritas.ac.cr

Xue, C., Wu, Q., Zhou, W., Yang, W. Y Story, D. (2006): "Comparison of chinase medicine education and training in China and Australia", Annals Academy of Medicine Singapore, $V$. $35, N^{\circ} 11$, p. 775-779, Singapore. 
